\title{
Some results for fourth-order nonlinear differential equation with singularity
}

Yun Xin $^{1 *}$ and Zhibo Cheng ${ }^{2}$

\section{*Correspondence:}

xy_1982@126.com

${ }^{1}$ College of Computer Science and

Technology, Henan Polytechnic

University, Jiaozuo, 454000, China

Full list of author information is

available at the end of the article

\begin{abstract}
By application of Green's function and some fixed point theorems, i.e., Leray-Schauder alternative principle and Schauder's fixed point theorem, we establish two new existence results of positive periodic solutions for a nonlinear fourth-order singular differential equation, which extend and improve significantly existing results in the literature.
\end{abstract}

MSC: 34B16; 34C25; 34B18

Keywords: fourth-order differential equation; Green's function; singularity; Leray-Schauder alternative principle; Schauder's fixed point theorem

\section{Introduction}

Generally speaking, differential equations with singularities have been considered from the very beginning of the discipline. The main reason is that singular forces are ubiquitous in applications, gravitational and electromagnetic forces being the most obvious examples. In 1965, Ding [1] discussed the Brillouin electron beam focusing system

$$
x^{\prime \prime}+a(1+\cos 2 t) x=\frac{1}{x},
$$

and obtained the existence of a positive periodic solution for the model if $0<a<\frac{1}{4}$.

Afterwards, in 1979, Taliaferro [2] discussed the model equation with singularity

$$
y^{\prime \prime}+\frac{q(t)}{y^{\alpha}}=0, \quad 0<t<1
$$

subject to

$$
y(0)=0=y(1)
$$

and obtained the existence of a solution for the problem. Here, $\alpha>0, q \in C(0,1)$ with $q>0$ on $(0,1)$ and $\int_{0}^{1} t(1-t) q(t) d t<\infty$. We call the equation with strong force condition if $\alpha \geq 1$ and we call it with weak force condition if $0<\alpha<1$.

Ding and Taliaferro's work has attracted the attention of many specialists in differential equations. More recently, the method of lower and upper solutions [3, 4], the Poincaré-

(c) 2015 Xin and Cheng. This article is distributed under the terms of the Creative Commons Attribution 4.0 International License (http://creativecommons.org/licenses/by/4.0/), which permits unrestricted use, distribution, and reproduction in any medium, provided you give appropriate credit to the original author(s) and the source, provide a link to the Creative Commons license, and indicate if changes were made. 
Birkhoff twist theorem [5-7], topological degree theory [8, 9], Schauder's fixed point theorem [10], the Krasnoselskii fixed point theorem in a cone [11, 12] and fixed point index theory [13] have been employed to investigate the existence of positive periodic solutions of singular second-order and third-order differential equations.

At the beginning, most of work concentrated on second-order and third-order singular differential equations, as in the references we mentioned above. Recently some results on fourth-order differential equations have been published (see [14-20]). In 2003, Conti et al. [15] studied the fourth-order equation

$$
u^{(4)}(t)-c u^{\prime \prime}(t)=f(t, u(t)), \quad t \in[0, T]
$$

with periodic boundary conditions, where $c \geq-(\pi / T)^{2}, f: \mathbb{R}^{2} \rightarrow \mathbb{R}$ is continuous, $T$-periodic in $t$ and has a superlinear behavior at 0 and at infinity. Under these assumptions, they showed that for each positive integer $n \geq 1$ the problem admits a $T$-periodic solution having precisely $2 n$ simple zeroes in $[0, T]$. The proof was inspired by Nehari's argument of combining variational methods and nodal properties of solutions. However, here a new and subtle min-max procedure is built, allowing one to interpret nodal properties of solutions of the problem as a topological property and to get these solutions by means of a variational principle with two constraints. In 2009, Li and Zhang [17] used some Sobolev constants to explicitly characterize a class of potentials $q(t) \in L^{p}(0, T)$ for which the periodic beam equation with periodic boundary condition

$$
\begin{aligned}
& u^{(4)}(t)=q(t) u(t), \quad t \in(0, T), \\
& u^{(i)}(0)=u^{(i)}(T), \quad 0 \leq i \leq 3,
\end{aligned}
$$

is non-degenerate. As an application, they obtained the uniqueness of periodic solutions of a certain class of superlinear beam equations. Recently, Mosconi and Santra [19] proved that if $F \in C(\mathbb{R})$ was coercive and $\left\{F^{\prime}=0\right\}$ was discrete, then the EFK equation

$$
u^{\prime \prime \prime \prime}-c u^{\prime \prime}+F^{\prime}(u)=0
$$

possesses $L^{\infty}(\mathbb{R})$ solutions if and only if $F^{\prime}$ changed sign at least twice. As a corollary they proved that if $u_{n}$ solved

$$
u_{n}^{\prime \prime \prime \prime}+c_{n}^{2} u_{n}^{\prime \prime}+F^{\prime}\left(u_{n}\right)=0
$$

then $\left\|u_{n}\right\|_{\infty} \rightarrow+\infty$ if $c_{n} \rightarrow 0$, provided $F$ has a unique local minimum, its only minimum is non-degenerate and $\operatorname{int}\left(\left\{F^{\prime}=0\right\}\right)=\varnothing$. Finally, they gave criteria ensuring existence and non-existence of $T$-periodic solutions to (1.2) when $F$ had multiple well.

In the above papers, the authors investigated fourth-order equations. However, the study on the singular fourth-order equation is relatively infrequent. Motivated by $[15,17,19]$, in this paper, we further consider a fourth-order nonlinear differential equation as follows:

$$
x^{(4)}(t)-\rho^{4} x(t)=f(t, x(t))+e(t)
$$

where $\rho \in R^{+}, e(t) \in L^{1}(R)$ is an $\omega$-periodic function, $f \in \operatorname{Car}\left(R \times R^{+}, R\right)$ is an $L^{2}$ Carathéodory function, i.e., it is measurable in the first variable and continuous in the 
second variable, and for every $0<r<s$, there exists $h_{r, s} \in L^{2}[0, \omega]$ such that $|f(t, x(t))| \leq h_{r, s}$ for all $x \in[r, s]$ and a.e. $t \in[0, \omega], f$ is an $\omega$-periodic function about $t$. The nonlinear term $f$ of (1.3) can be with a singularity at origin, i.e.,

$$
\lim _{x \rightarrow 0^{+}} f(t, x)=+\infty \quad\left(\text { or } \lim _{x \rightarrow 0^{+}} f(t, x)=-\infty\right), \quad \text { uniformly in } t .
$$

It is said that (1.3) is of repulsive type (resp. attractive type) if $f(t, x) \rightarrow+\infty$ (resp. $f(t, x) \rightarrow$ $-\infty)$ as $x \rightarrow 0^{+}$.

The remaining part of the paper is organized as follows. In Section 2, the Green's function for the fourth-order linear differential equation

$$
x^{(4)}(t)-\rho^{4} x(t)=h(t)
$$

will be given. Here, $h \in C(R,(0,+\infty))$ is an $\omega$-periodic function. Some useful properties for Green's function are obtained. In Section 3, by employing Green's function and a nonlinear alternative principle of Leray-Schauder, we state and prove the first existence result for (1.3). The result is applicable to the case of a strong singularity as well as the case of a weak singularity. In Section 4, we get a second existence result for (1.3). We prove that a weak singularity enables the achievement of new existence criteria through a basic application of Schauder's fixed point theorem.

To conclude this introduction, some notations are presented as follows: for a.e. means for almost every. We write $d(t) \succ 0$ if $d(t) \geq 0$ for a.e. $t \in[0, \omega]$ and it is positive in a set of positive measure. The set of positive real numbers is denoted by $R^{+}$. For a given function $e \in L^{1}[0, \omega]$, we denote the essential supremum and infimum by $e^{*}$ and $e_{*}$ if they exist. Define $C_{\omega}=\{x(t) \in C(R, R): x(t+\omega)=x(t)\}$. Let $X=\{\phi \in C(\mathbb{R}, \mathbb{R}): \phi(t+\omega)=\phi(t)\}$ with the maximum norm $\|\phi\|=\max _{0 \leq t \leq \omega}|\phi(t)|$. Obviously, $X$ is a Banach space.

\section{Green's function of fourth-order differential equation}

Lemma 2.1 For $\rho>0$ and $h \in X$, the equation

$$
\left\{\begin{array}{l}
u^{(4)}-\rho^{4} u=h(t) \\
u^{(i)}(0)=u^{(i)}(\omega), \quad i=0,1,2,3
\end{array}\right.
$$

has a unique solution which is of the form

$$
u(t)=\int_{0}^{\omega} G(t, s)(-h(s)) d s
$$

where

$$
G(t, s)= \begin{cases}\frac{\exp (\rho(t-s))+\exp (\rho(s+\omega-t))}{4 \rho^{3}(\exp (\rho \omega)-1)}+\frac{\sin \rho(t-s)-\sin \rho(t-s-\omega)}{4 \rho^{3}(1-\cos \rho \omega)}, & 0 \leq s \leq t \leq \omega, \\ \frac{\exp (\rho(t+\omega-s))+\exp (\rho(s-t))}{4 \rho^{3}(\exp (\rho \omega)-1)}+\frac{\sin \rho(s-t)-\sin \rho(s-\omega-t)}{4 \rho^{3}(1-\cos \rho \omega)}, & 0 \leq t \leq s \leq \omega .\end{cases}
$$

Proof It is easy to check that the associated homogeneous equation of (2.1) has the solution

$$
v(t)=c_{1} \exp (\rho t)+c_{2} \exp (-\rho t)+c_{3} \cos \rho t+c_{4} \sin \rho t
$$


Applying the method of variation of parameters, we get

$$
\begin{array}{ll}
c_{1}^{\prime}(t)=\frac{\exp (-\rho t) h(t)}{4 \rho^{3}}, & c_{2}^{\prime}(t)=\frac{-\exp (\rho t) h(t)}{4 \rho^{3}}, \\
c_{3}^{\prime}(t)=\frac{\sin (\rho t) h(t)}{2 \rho^{3}}, & c_{4}^{\prime}(t)=\frac{-\cos (\rho t) h(t)}{2 \rho^{3}},
\end{array}
$$

and then

$$
\begin{array}{ll}
c_{1}(t)=c_{1}(0)+\int_{0}^{t} \frac{\exp (-\rho s) h(s)}{4 \rho^{3}} d s, & c_{2}(t)=c_{2}(0)+\int_{0}^{t}-\frac{\exp (\rho s) h(s)}{4 \rho^{3}} d s, \\
c_{3}(t)=c_{3}(0)+\int_{0}^{t} \frac{\sin (\rho s) h(s)}{2 \rho^{3}} d s, & c_{4}(t)=c_{4}(0)+\int_{0}^{t}-\frac{\cos (\rho s) h(s)}{2 \rho^{3}} d s .
\end{array}
$$

Noting that $u(0)=u(\omega), u^{\prime}(0)=u^{\prime}(\omega), u^{\prime \prime}(0)=u^{\prime \prime}(\omega), u^{\prime \prime \prime}(0)=u^{\prime \prime \prime}(\omega)$, we obtain

$$
\begin{aligned}
& c_{1}(0)=\int_{0}^{\omega} \frac{\exp (\rho(\omega-s))}{4 \rho^{3}(1-\exp (\rho \omega))} h(s) d s, \\
& c_{2}(0)=\int_{0}^{\omega} \frac{\exp (\rho s)}{4 \rho^{3}(1-\exp (\rho \omega))} h(s) d s, \\
& c_{3}(0)=-\int_{0}^{\omega} \frac{\sin (\rho s)-\sin (\rho(s-\omega))}{4 \rho^{3}(1-\cos \rho \omega)} h(s) d s, \\
& c_{4}(0)=-\int_{0}^{\omega} \frac{\cos (\rho(s-\omega))-\cos (\rho s)}{4 \rho^{3}(1-\cos \rho \omega)} h(s) d s .
\end{aligned}
$$

Therefore

$$
\begin{aligned}
u(t)= & c_{1}(t) \exp (\rho t)+c_{2}(t) \exp (-\rho t)+c_{3}(t) \cos \rho t+c_{4}(t) \sin \rho t \\
= & \int_{0}^{t}\left\{\frac{\exp (\rho(t-s))+\exp (\rho(s+\omega-t))}{4 \rho^{3}(\exp (\rho \omega)-1)}+\frac{\sin \rho(t-s)-\sin \rho(t-s-\omega)}{4 \rho^{3}(1-\cos \rho \omega)}\right\}(-h(s)) d s \\
& +\int_{t}^{\omega}\left\{\frac{\exp (\rho(t+\omega-s))+\exp (\rho(s-t))}{4 \rho^{3}(\exp (\rho \omega)-1)}+\frac{\sin \rho(s-t)-\sin \rho(s-t-\omega)}{4 \rho^{3}(1-\cos \rho \omega)}\right\} \\
& \times(-h(s)) d s \\
= & \int_{0}^{\omega} G(t, s)(-h(s)) d s,
\end{aligned}
$$

where $G(t, s)$ is defined as in (2.3).

By a direct calculation, we get that the solution $u$ satisfies the periodic boundary value condition of problem (2.1).

Now we present the properties of Green's functions for (2.1).

Lemma 2.2 $\int_{0}^{\omega} G(t, s) d s=\frac{1}{\rho^{4}}$ and if $\rho<\frac{\pi}{\omega}$ holds, then $0<l<G(t, s) \leq L$ for all $t \in[0, \omega]$ and $s \in[0, \omega]$.

Proof From (2.3) we can get $\int_{0}^{\omega} G(t, s) d s=\frac{1}{\rho^{4}}$. If $\rho<\frac{\pi}{\omega}$, we get $G(t, s)>0$ for all $t \in[0, \omega]$ and $s \in[0, \omega]$. 
Next we compute a lower and an upper bound for $G(t, s)$ for $s \in[0, \omega]$. We have

$$
l:=\frac{\exp \left(\frac{\rho \omega}{2}\right)}{2 \rho^{3}(\exp (\rho \omega)-1)} \leq G(t, s)<\frac{1+\exp (\rho \omega)}{4 \rho^{3}(\exp (\rho \omega)-1)}+\frac{1}{2 \rho^{3}(1-\cos \rho \omega)}:=L,
$$

and the proof is complete.

\section{Existence results (I)}

In the section, we state and prove the first existence results. The proof is based on the following nonlinear alternative of Leray-Schauder, which can be found in [21].

Lemma 3.1 Let $C$ be a convex subset of a normed linear space $E$, and let $U$ be an open subset of $C$ with $0 \in U$. Then every compact, continuous map $F: \bar{U} \rightarrow C$ has at least one of the following properties:

(I) F has a fixed point in $\bar{U}$ or

(II) there is $a u \in \partial U$ and $\lambda \in(0,1)$ with $x=\lambda F x$.

Define the function $\gamma: R \rightarrow R$ by

$$
\gamma(t)=\int_{0}^{\omega} G(t, s) e(s) d s
$$

which under the hypothesis in Section 2 is the unique $\omega$-periodic solution of

$$
x^{(4)}(t)-\rho^{4} x(t)=e(t)
$$

Under Lemma 2.2, we always denote

$$
\sigma=l / L
$$

Obviously, we have $0<\sigma<1$.

We take $X=C_{\omega}$ with $\|x\|=\max _{t}|x(t)|$. Define the operator $T: X \rightarrow X$,

$$
(T x)(t)=\int_{0}^{\omega} G(t, s) f(s, x(s)+\gamma(s)) d s .
$$

Define the cone $K$ in $X$ by

$$
K=\left\{x \in X: x(t) \geq 0 \text { for all } t \in R \text { and } \min _{t \in R} x(t) \geq \sigma\|x\|\right\} .
$$

Theorem 3.1 Assume that $\rho<\frac{\pi}{\omega}$ holds. Suppose that the following conditions are satisfied:

$\left(\mathrm{H}_{1}\right)$ For each constant $L>0$, there exists a continuous function $\phi_{L} \succ 0$ such that $f(t, x) \geq$ $\phi_{L}(t)$ for a.e. $t$ and $x \in(0, L]$.

$\left(\mathrm{H}_{2}\right)$ There exist continuous, non-negative functions $g(x), h(x)$ and $k(t)$ such that

$$
0 \leq f(t, x) \leq k(t)(g(x)+h(x)) \quad \text { for all } x \in(0, \infty) \text { and a.e. } t
$$

and $g(x)>0$ is non-increasing, $h(x) / g(x)$ is non-decreasing in $x$. 
$\left(\mathrm{H}_{3}\right)$ There exists a positive number $r>0$ such that

$$
\frac{r}{g\left(\sigma r+\gamma_{*}\right)\left(1+\frac{h\left(r+\gamma^{*}\right)}{g\left(r+\gamma^{*}\right)}\right)}>K^{*}
$$

where $K(t)=\int_{0}^{\omega} G(t, s) k(s) d s$.

If $\gamma_{*} \geq 0$, then (1.3) has at least one positive $\omega$-periodic solution $x$ with $x(t)>\gamma(t)$ for all $t$ and $0<\|x-\gamma\|<r$.

Proof Step 1. Consider the equation

$$
x^{(4)}(t)-\rho^{4} x(t)=f(t, x(t)+\gamma(t))
$$

It is easy to see that if (3.2) has a positive $\omega$-periodic solution $x$ satisfying $x(t)+\gamma(t)>0$ for $t \in[0, \omega]$ and $0<\|x\|<r$, then $u(t)=x(t)+\gamma(t)$ is a positive $\omega$-periodic solution of (1.3) with $0<\|u-\gamma\|<r$. So we only need to consider (3.2).

Step 2. In order to study (3.2), we first consider a family of equations.

Since $\left(\mathrm{H}_{3}\right)$ holds, we can choose $n_{0} \in\{1,2, \ldots\}$ such that $\frac{1}{n_{0}}<\sigma r+\gamma_{*}$ and

$$
K^{*} g\left(\sigma r+\gamma_{*}\right)\left(1+\frac{h\left(r+\gamma^{*}\right)}{g\left(r+\gamma^{*}\right)}\right)+\frac{1}{n_{0}}<r
$$

Let $N_{0}=\left\{n_{0}, n_{0}+1, \ldots\right\}$. Fix $n \in N_{0}$. Consider the family of equations

$$
x^{(4)}(t)-\rho^{4} x(t)=\mu f_{n}(t, x(t)+\gamma(t))+\frac{\rho^{4}}{n},
$$

where $\mu \in[0,1]$, and

$$
f_{n}(t, x)= \begin{cases}f(t, x) & \text { if } x \geq \frac{1}{n} \\ f\left(t, \frac{1}{n}\right) & \text { if } x \leq \frac{1}{n}\end{cases}
$$

Now we prove that (3.3) has a periodic solution for each $n$.

If $x$ is a periodic solution of problem (3.3), we have from Lemma 2.1 that

$$
x(t)=\mu \int_{0}^{\omega} G(t, s) f_{n}(t, x(s)+\gamma(s)) d s+\frac{1}{n} .
$$

Define $T_{n}: K \rightarrow X$ by

$$
\left(T_{n} x\right)(t)=\int_{0}^{\omega} G(t, s) f_{n}(t, x(s)+\gamma(s)) d s
$$

So, solving (3.3) is equivalent to the following fixed point problem:

$$
x(t)=\mu\left(T_{n} x\right)(t)+\frac{1}{n} .
$$


Let $\Omega=\{x \in K \mid\|x\|<r\}$. We claim $T_{n}(\Omega) \subset K$. In fact, $\forall x \in K$, we have

$$
\begin{aligned}
\min _{t \in R}\left(T_{n} x\right)(t) & =\min _{t \in R} \int_{0}^{\omega} G(t, s) f_{n}(s, x(s)+\gamma(s)) d s \\
& =\min _{t \in R} \int_{0}^{\omega} G(t, s) f_{n}^{+}(s, x(s)+\gamma(s)) d s \\
& \geq m \int_{0}^{\omega} f_{n}^{+}(s, x(s)+\gamma(s)) d s \\
& =\sigma M \int_{0}^{\omega} f_{n}^{+}(s, x(s)+\gamma(s)) d s \\
& \geq \sigma \max _{t \in R} \int_{0}^{\omega} G(t, s) f_{n}^{+}(s, x(s)+\gamma(s)) d s \\
& =\sigma \max _{t \in R} \int_{0}^{\omega} G(t, s) f_{n}(s, x(s)+\gamma(s)) d s \\
& =\sigma\left\|T_{n} x\right\|,
\end{aligned}
$$

here $f_{n}^{+}(t, x)=\max \left\{0, f_{n}(t, x)\right\}$. This implies that $T_{n}(\Omega) \subset K$. Besides, it is easy to see that $T_{n}: \Omega \rightarrow K$ is completely continuous.

We claim that any fixed point $x$ of (3.4) for all $\mu \in[0,1]$ must satisfy $\|x\| \neq r$. Otherwise, assume that $x$ is a fixed point of (3.4) for some $\mu \in[0,1]$ such that $\|x\|=r$. Thus, we have

$$
\begin{aligned}
x(t)-\frac{1}{n} & =\mu \int_{0}^{\omega} G(t, s) f_{n}(s, x(s)+\gamma(s)) d s \\
& =\mu \int_{0}^{\omega} G(t, s) f_{n}^{+}(s, x(s)+\gamma(s)) d s \\
& \geq \mu m \int_{0}^{\omega} f_{n}^{+}(s, x(s)+\gamma(s)) d s \\
& =\mu \sigma M \int_{0}^{\omega} f_{n}^{+}(s, x(s)+\gamma(s)) d s \\
& \geq \sigma \max _{t \in R}\left\{\mu \int_{0}^{\omega} G(t, s) f_{n}^{+}(s, x(s)+\gamma(s)) d s\right\} \\
& =\sigma \max _{t \in R}\left\{\mu \int_{0}^{\omega} G(t, s) f_{n}(s, x(s)+\gamma(s)) d s\right\} \\
& =\sigma\left\|x-\frac{1}{n}\right\| .
\end{aligned}
$$

Therefore, we have

$$
x(t) \geq \sigma\left\|x-\frac{1}{n}\right\|+\frac{1}{n} \geq \sigma\left(\|x\|-\frac{1}{n}\right)+\frac{1}{n} \geq \sigma r .
$$

So, we have

$$
x(t)+\gamma(t) \geq \sigma r+\gamma_{*}>\frac{1}{n}
$$


since $\frac{1}{n} \leq \frac{1}{n_{0}}<\sigma r+\gamma_{*}$. Thus, from $\left(\mathrm{H}_{2}\right)$ we have

$$
\begin{aligned}
x(t) & =\mu \int_{0}^{\omega} G(t, s) f_{n}(s, x(s)+\gamma(s)) d s+\frac{1}{n} \\
& =\mu \int_{0}^{\omega} G(t, s) f_{n}^{+}(s, x(s)+\gamma(s)) d s+\frac{1}{n} \\
& =\mu \int_{0}^{\omega} G(t, s) f^{+}(s, x(s)+\gamma(s)) d s+\frac{1}{n} \\
& \leq \int_{0}^{\omega} G(t, s) f^{+}(s, x(s)+\gamma(s)) d s+\frac{1}{n} \\
& \leq \int_{0}^{\omega} G(t, s) k(s) g(x(s)+\gamma(s))\left(1+\frac{h(x(s)+\gamma(s))}{g(x(s)+\gamma(s))}\right) d s+\frac{1}{n} \\
& \leq g\left(\sigma r+\gamma_{*}\right)\left(1+\frac{h\left(r+\gamma^{*}\right)}{g\left(r+\gamma^{*}\right)}\right) K^{*}+\frac{1}{n_{0}}
\end{aligned}
$$

where $f^{+}(t, x)=\max \{0, f(t, x)\}$.

Therefore,

$$
r=\|x\| \leq g\left(\sigma r+\gamma_{*}\right)\left(1+\frac{h\left(r+\gamma^{*}\right)}{g\left(r+\gamma^{*}\right)}\right) K^{*}+\frac{1}{n_{0}} .
$$

This is a contradiction to the choice of $n_{0}$ and the claim is proved.

From this claim, Lemma 3.1 guarantees that

$$
x(t)=\left(T_{n} x\right)(t)+\frac{1}{n}
$$

has a fixed point, denoted by $x_{n}$, in $\Omega$, i.e.,

$$
x^{(4)}(t)-\rho^{4} x(t)=f_{n}(t, x(t)+\gamma(t))+\frac{\rho^{4}}{n}
$$

has an $\omega$-periodic solution $x_{n}$ with $\left\|x_{n}\right\|<r$. Since $x_{n}(t) \geq \frac{1}{n}>0$ for all $t \in[0, \omega], x_{n}$ is actually a positive $\omega$-periodic solution of (3.6).

Now we show that $x_{n}(t)+\gamma(t)$ has a uniform positive lower bound, i.e., there exists a constant $\vartheta>0$, independent of $n \in N_{0}$, such that

$$
\min _{t \in[0, \omega]}\left\{x_{n}(t)+\gamma(t)\right\} \geq \vartheta
$$

for all $n \in N_{0}$. To see this, we know from $\left(\mathrm{H}_{1}\right)$ that there exists a continuous function $\phi_{r+\gamma^{*}}(t)>0$ such that $f(t, x) \geq \phi_{r+\gamma^{*}}(t)$ for a.e. $t$ and $0<x \leq r+\gamma^{*}$. Let $x_{r+\gamma^{*}}(t)$ be the unique $\omega$-periodic solution to

$$
x^{(4)}(t)-\rho^{4} x(t)=\phi_{r+\gamma^{*}}(t)
$$

then we have

$$
x_{r+\gamma^{*}}(t)+\gamma(t)=\int_{0}^{\omega} G(t, s) \phi_{r+\gamma^{*}}(s) d s+\gamma(t) \geq \Phi_{*}+\gamma_{*}>0,
$$

where $\Phi(t)=\int_{0}^{\omega} G(t, s) \phi_{r+\gamma^{*}}(s) d s$. 
Since $x_{n}(t)+\gamma(t) \leq r+\gamma^{*}$ and $x_{n}+\gamma_{*} \geq \frac{1}{n}$, we have

$$
\begin{aligned}
x_{n}(t)+\gamma(t) & =\int_{0}^{\omega} G(t, s) f_{n}\left(s, x_{n}(s)+\gamma(s)\right) d s+\gamma(t)+\frac{1}{n} \\
& =\int_{0}^{\omega} G(t, s) f^{+}\left(s, x_{n}(s)+\gamma(s)\right) d s+\gamma(t)+\frac{1}{n} \\
& \geq \int_{0}^{\omega} G(t, s) \phi_{r+\gamma^{*}}(s) d s+\gamma(t) \\
& \geq \Phi_{*}+\gamma_{*}:=\vartheta
\end{aligned}
$$

So we have $x_{n}(t)+\gamma(t) \geq \vartheta$ for all $n$.

Step 3. In order to pass the solutions $x_{n}$ of (3.6) to that of the original problem (3.2), we need to show that $\left\{x_{n}\right\}_{n \in N_{0}}$ is compact.

First we show

$$
\left\|x_{n}^{\prime}\right\| \leq M_{1}
$$

for some constant $M_{1}>0$ (independent of $n \in N_{0}$ ) and for all $n \in N_{0}$.

In fact, since $x_{n}$ is an $\omega$-periodic solution of (3.6), we have

$$
x_{n}^{(4)}(t)-\rho^{4} x_{n}(t)=f_{n}\left(t, x_{n}(t)+\gamma(t)\right)+\frac{\rho^{4}}{n} .
$$

Multiplying both sides of (3.9) by $x_{n}(t)$ and integrating from 0 to $\omega$, we have

$$
\begin{aligned}
& \int_{0}^{\omega} x_{n}^{(4)}(t) x_{n}(t) d t+\rho^{4} \int_{0}^{\omega}\left|x_{n}(t)\right|^{2} d t \\
& \quad=\int_{0}^{\omega} f_{n}\left(t, x_{n}(t)+\gamma(t)\right) x_{n}(t) d t+\frac{\rho^{4}}{n} \int_{0}^{\omega} x_{n}(t) d t
\end{aligned}
$$

Substituting $\int_{0}^{\omega} x_{n}^{(4)}(t) x_{n}(t) d t=\int_{0}^{\omega}\left|x_{n}^{\prime \prime}(t)\right|^{2} d t$ into (3.10), we have

$$
\begin{aligned}
\int_{0}^{\omega}\left|x_{n}^{\prime \prime}(t)\right|^{2} d t & =\rho^{4} \int_{0}^{\omega}\left|x_{n}(t)\right|^{2} d t+\int_{0}^{\omega} f_{n}\left(t, x_{n}(t)+\gamma(t)\right) x_{n}(t) d t+\frac{\rho^{4}}{n} \int_{0}^{\omega} x_{n}(t) d t \\
& \leq \rho^{4} \int_{0}^{\omega}\left|x_{n}(t)\right|^{2} d t+\int_{0}^{\omega}\left|f_{n}\left(t, x_{n}(t)+\gamma(t)\right)\right|\left|x_{n}(t)\right| d t+\frac{\rho^{4}}{n} \int_{0}^{\omega}\left|x_{n}(t)\right| d t \\
& \leq \rho^{4} r^{2} \omega+\left(\int_{0}^{\omega}\left|f_{n}\left(t, x_{n}(t)+\gamma(t)\right)\right|^{2} d t\right)^{\frac{1}{2}}\left(\int_{0}^{\omega}\left|x_{n}(t)\right|^{2} d t\right)^{\frac{1}{2}}+\frac{\rho^{4}}{n_{0}} r \omega \\
& \leq \rho^{4} r^{2} \omega+r \sqrt{\omega}\left|f_{r}\right|_{2}+\frac{|d|}{n_{0}} r \omega
\end{aligned}
$$

where $\left|f_{r}\right|=\max _{\vartheta \leq x_{n}}(t)+\gamma \leq r+\gamma^{*}\left|f_{n}\left(t, x_{n}(t)+\gamma(t)\right)\right|,\left|f_{r}\right|_{2}=\left(\int_{0}^{\omega}\left|f_{r}\right|^{2} d t\right)^{\frac{1}{2}}$. It is easy to see that there exists a constant $M_{1}^{\prime}>0$ such that

$$
\int_{0}^{\omega}\left|x_{n}^{\prime \prime}(t)\right|^{2} d t \leq M_{1}^{\prime}
$$


From $x_{n}(0)=x_{n}(\omega)$ we know that there exists a point $t_{0} \in[0, \omega]$ such that $x_{n}^{\prime}\left(t_{0}\right)=0$. Therefore, we have

$$
\left\|x_{n}^{\prime}\right\|=\max _{t}\left|\int_{t_{0}}^{t} x_{n}^{\prime \prime}(s) d s\right| \leq \int_{0}^{\omega}\left|x_{n}^{\prime \prime}(s)\right| d s \leq \omega^{\frac{1}{2}}\left(\int_{0}^{\omega}\left|x_{n}^{\prime \prime}(s)\right|^{2} d s\right)^{\frac{1}{2}} \leq \omega^{\frac{1}{2}} M_{1}^{\prime \frac{1}{2}}:=M_{1} .
$$

The fact $\left\|x_{n}\right\|<r$ and (3.8) show that $\left\{x_{n}\right\}_{n \in N_{0}}$ is bounded and an equi-continuous family on $R$. Now the Arzela-Ascoli theorem guarantees that $\left\{x_{n}\right\}_{n \in N_{0}}$ has a subsequence, $\left\{x_{n_{k}}\right\}_{k \in N}$, converging uniformly on $R$ to a function $x \in X$. From the fact $\left\|x_{n}\right\|<r$ and $\vartheta \leq x_{n}+\gamma, x$ satisfies $\vartheta \leq x(t)+\gamma(t) \leq r+\gamma^{*}$ for all $t$. Moreover, $x_{n_{k}}$ satisfies the integral equation

$$
x_{n_{k}}(t)=\int_{0}^{\omega} G(t, s) f\left(s, x_{n_{k}}(s)+\gamma(s)\right) d s+\frac{1}{n_{k}} \text {. }
$$

Letting $k \rightarrow \infty$, we get

$$
x(t)=\int_{0}^{\omega} G(t, s) f(s, x(s)+\gamma(s)) d s
$$

Therefore, $x$ is a positive periodic solution of (3.2) and satisfies $0<\|x\| \leq r$. Besides, it is not difficult to show that $\|x\|<r$ by noting that if $\|x\|=r$, the argument similar to the proof of the first claim will yield a contradiction.

Combining the three steps, the proof is completed.

Corollary 3.1 Assume that $\rho<\frac{\pi}{\omega}$ holds. Suppose that the following condition is satisfied:

$\left(\mathrm{F}_{1}\right)$ There exist continuous functions $d(t), \hat{d}(t) \succ 0$ and $\tau>0,0 \leq \eta<1$, such that

$$
0 \leq \frac{\hat{d}(t)}{x^{\tau}} \leq f(t, x) \leq \frac{d(t)}{x^{\tau}}+d(t) x^{\eta} \quad \text { for all } x>0 \text { and a.e. } t
$$

If $\gamma_{*} \geq 0$, then (1.3) has at least one positive $\omega$-periodic solution.

Proof We will apply Theorem 3.1. We take

$$
\phi_{L}(t)=\frac{\hat{d}(t)}{L^{\tau}}, \quad k(t)=d(t), \quad g(x)=\frac{1}{x^{\tau}}, \quad h(x)=x^{\eta}
$$

Then $\left(\mathrm{H}_{1}\right)$ and $\left(\mathrm{H}_{2}\right)$ are satisfied and the existence condition $\left(\mathrm{H}_{3}\right)$ becomes

$$
\frac{r\left(\sigma r+\gamma_{*}\right)^{\tau}}{1+\left(r+\gamma^{*}\right)^{\tau+\eta}}>\Psi^{*}
$$

where $\Psi(t)=\int_{0}^{\omega} G(t, s) d(s) d s$ for some $r>0$.

Since $\tau>0,0 \leq \eta<1$ and $\gamma_{*} \geq 0$, we can choose $r>0$ large enough such that (3.11) is satisfied.

Theorem 3.2 Assume that $\rho<\frac{\pi}{\omega}$ and $\left(\mathrm{H}_{2}\right)-\left(\mathrm{H}_{3}\right)$ hold. If $\gamma_{*}>0$, then (1.3) has at least one positive $\omega$-periodic solution $x$ with $x(t)>\gamma(t)$ for all $t$ and $0<\|x-\gamma\|<r$. 
Proof We will follow the same strategy and notations as in the proof of Theorem 3.1. Step 1 and Step 2 are the same as in the proof of Theorem 3.1. Now, we consider that $x_{n}(t)+\gamma(t)$ has a uniform positive lower bound, i.e., there exists a constant $\vartheta_{1}>0$, independent of $n \in N_{0}$, such that

$$
\min _{t \in[0, \omega]}\left\{x_{n}(t)+\gamma(t)\right\} \geq \vartheta_{1}
$$

for all $n \in N_{0}$.

Since $x_{n}+\gamma>\frac{1}{n}$ and $\gamma_{*}>0$, from Lemma 2.2, we know that $G$ and $f$ are of non-negative sign. Thus we have

$$
\begin{aligned}
x_{n}(t)+\gamma(t) & =\int_{0}^{\omega} G(t, s) f_{n}\left(s, x_{n}(s)+\gamma(s)\right) d s+\gamma(t)+\frac{1}{n} \\
& =\int_{0}^{\omega} G(t, s) f\left(s, x_{n}(s)+\gamma(s)\right) d s+\gamma(t)+\frac{1}{n} \\
& \geq \int_{0}^{\omega} G(t, s) f\left(s, x_{n}(s)+\gamma(s)\right) d s+\gamma(t) \\
& \geq \gamma_{*}:=\vartheta_{1} .
\end{aligned}
$$

So we have $x_{n}(t)+\gamma(t) \geq \vartheta_{1}$.

The proof left is the same as in Theorem 3.1.

Corollary 3.2 Assume that $\rho<\frac{\pi}{\omega}$ holds. Suppose that the following condition is satisfied:

$\left(\mathrm{F}_{2}\right)$ There exists a continuous function $d(t) \geq 0$ for a.e. $t \in[0, \omega]$ and $\tau>0,0 \leq \eta<1$, such that

$$
0 \leq f(t, x) \leq \frac{d(t)}{x^{\tau}}+d(t) x^{\eta} \quad \text { for all } x>0 \text {, for a.e.t. }
$$

If $\gamma_{*}>0$, then (1.3) has at least one positive $\omega$-periodic solution.

Proof We will apply Theorem 3.2. Take

$$
k(t)=d(t), \quad g(x)=\frac{1}{x^{\tau}}, \quad h(x)=x^{\eta},
$$

then $\left(\mathrm{H}_{2}\right)$ is satisfied and the existence condition $\left(\mathrm{H}_{3}\right)$ becomes

$$
\frac{r\left(\sigma r+\gamma_{*}\right)^{\tau}}{1+\left(r+\gamma^{*}\right)^{\tau+\eta}}>\Psi^{*}
$$

where $\Psi(t)=\int_{0}^{\omega} G(t, s) d(s) d s$ for some $r>0$.

Since $\tau>0,0 \leq \eta<1$ and $\gamma_{*}>0$, we can choose $r>0$ large enough such that (3.12) is satisfied.

Theorem 3.3 Assume that $\rho<\frac{\pi}{\omega}$ and $\left(\mathrm{H}_{1}\right)-\left(\mathrm{H}_{3}\right)$ hold. And the following condition is satisfied:

$\left(\mathrm{H}_{4}\right) \gamma_{*}+\Phi_{*}>0$, here $\Phi(t)=\int_{0}^{\omega} G(t, s) \phi_{r+\gamma^{*}}(s) d s$. 
Then (1.3) has at least one positive $\omega$-periodic solution $x$ with $x(t)>\gamma(t)$ for all $t$ and $0<$ $\|x-\gamma\|<r$.

Proof We will follow the same strategy and notations as in the proof of Theorem 3.1. Step 1 and Step 2 are the same as in the proof of Theorem 3.1. Now, we mainly consider that $x_{n}(t)+\gamma(t)$ has a uniform positive lower bound, i.e., there exists a constant $\vartheta_{2}>0$, independent of $n \in N_{0}$, such that

$$
\min _{t \in[0, \omega]}\left\{x_{n}(t)+\gamma(t)\right\} \geq \vartheta_{2}
$$

for all $n \in N_{0}$.

Since $\left(\mathrm{H}_{1}\right)$ holds, we know that there exists a continuous function $\phi_{r+\gamma^{*}}(t)>0$ such that $f(t, x) \geq \phi_{r+\gamma^{*}}(t)$ for a.e. $t$ and $0<x \leq r+\gamma^{*}$. Let $x_{r+\gamma^{*}}(t)$ be the unique $\omega$-periodic solution to

$$
x^{(4)}(t)-\rho^{4} x(t)=\phi_{r+\gamma^{*}}(t)
$$

From $\left(\mathrm{H}_{4}\right)$ we have

$$
x_{r+\gamma^{*}}(t)+\gamma(t)=\int_{0}^{\omega} G(t, s) \phi_{r+\gamma^{*}}(s) d s+\gamma(t) \geq \Phi_{*}+\gamma_{*}>0 .
$$

Since $x_{n}(t)+\gamma(t) \leq r+\gamma^{*}$ and $x_{n}+\gamma_{*} \geq \frac{1}{n}$, then

$$
\begin{aligned}
x_{n}(t)+\gamma(t) & =\int_{0}^{\omega} G(t, s) f_{n}\left(s, x_{n}(s)+\gamma(s)\right) d s+\gamma(t)+\frac{1}{n} \\
& =\int_{0}^{\omega} G(t, s) f\left(s, x_{n}(s)+\gamma(s)\right) d s+\gamma(t)+\frac{1}{n} \\
& \geq \int_{0}^{\omega} G(t, s) \phi_{r+\gamma^{*}}(s) d s+\gamma(t) \\
& \geq \Phi_{*}+\gamma_{*}:=\vartheta_{2},
\end{aligned}
$$

i.e., $x_{n}(t)+\gamma(t) \geq \vartheta_{2}$.

The proof left is the same as in Theorem 3.1.

As an application of Theorem 3.3, we consider the case $\gamma_{*}=0$. The following Corollary 3.3 is a direct result of Theorem 3.3.

Corollary 3.3 Assume that $\rho<\frac{\pi}{\omega}$ and $\left(\mathrm{H}_{1}\right)-\left(\mathrm{H}_{3}\right)$ hold. If $\gamma_{*}=0$, then (1.3) has at least one positive $\omega$-periodic solution.

Theorem 3.4 Assume that $\rho<\frac{\pi}{\omega}$ and $\left(\mathrm{H}_{1}\right)-\left(\mathrm{H}_{3}\right)$ hold. And the following condition is satisfied:

$\left(\mathrm{H}_{5}\right) \gamma_{*}+\Phi_{*}^{\prime}>0$, here $\Phi^{\prime}(t)=\int_{0}^{\omega} G(t, s) \phi_{r}(s) d s$.

If $\gamma^{*} \leq 0$, then (1.3) has at least one positive $\omega$-periodic solution $x$ with $x(t)>\gamma(t)$ for all $t$ and $0<\|x-\gamma\|<r$. 
Proof We will follow the same strategy and notations as in the proof of Theorem 3.1. Step 1 and Step 2 are the same as in the proof of Theorem 3.1. Now, we mainly consider that $x_{n}(t)+\gamma(t)$ has a uniform positive lower bound, i.e., there exists a constant $\vartheta_{3}>0$, independent of $n \in N_{0}$, such that

$$
\min _{t \in[0, \omega]}\left\{x_{n}(t)+\gamma(t)\right\} \geq \vartheta_{3}
$$

for all $n \in N_{0}$.

Since $\left(\mathrm{H}_{1}\right)$ and $\gamma^{*} \leq 0$, we know that there exists a continuous function $\phi_{r}(t)>0$ such that $f(t, x) \geq \phi_{r}(t)$ for a.e. $t$ and $0<x \leq r+\gamma^{*} \leq r$. Let $x_{r}(t)$ be the unique $\omega$-periodic solution to

$$
x^{(4)}(t)-\rho^{4} x(t)=\phi_{r}(t)
$$

From $\left(\mathrm{H}_{5}\right)$ then we have

$$
x_{r}(t)+\gamma(t)=\int_{0}^{\omega} G(t, s) \phi_{r}(s) d s+\gamma(t) \geq \Phi_{*}^{\prime}+\gamma_{*}>0 .
$$

Since $x_{n}(t)+\gamma(t) \leq r+\gamma^{*} \leq r$ and $x_{n}+\gamma_{*} \geq \frac{1}{n}$, we have

$$
\begin{aligned}
x_{n}(t)+\gamma(t) & =\int_{0}^{\omega} G(t, s) f_{n}\left(s, x_{n}(s)+\gamma(s)\right) d s+\gamma(t)+\frac{1}{n} \\
& =\int_{0}^{\omega} G(t, s) f\left(s, x_{n}(s)+\gamma(s)\right) d s+\gamma(t)+\frac{1}{n} \\
& \geq \int_{0}^{\omega} G(t, s) \phi_{r}(s) d s+\gamma(t) \\
& \geq \Phi_{*}^{\prime}+\gamma_{*}:=\vartheta_{3} .
\end{aligned}
$$

So we have $x_{n}(t)+\gamma(t) \geq \vartheta_{3}$.

The proof left is the same as in Theorem 3.1.

Corollary 3.4 Assume that $\rho<\frac{\pi}{\omega}$ and $\left(\mathrm{F}_{1}\right)$ hold. If $\gamma^{*} \leq 0$ and $\gamma_{*}>-\frac{\hat{\Psi}_{*}}{r^{*}}$, here $\hat{\Psi}=$ $\int_{0}^{\omega} G(t, s) \hat{d}(s) d s$, then (1.3) has at least one positive $\omega$-periodic solution.

Proof We will apply Theorem 3.4. Take

$$
\phi_{L}(t)=\frac{\hat{d}(t)}{L^{\tau}}, \quad k(t)=d(t), \quad g(x)=\frac{1}{x^{\tau}}, \quad h(x)=x^{\eta},
$$

then $\left(\mathrm{H}_{1}\right)$ and $\left(\mathrm{H}_{2}\right)$ are satisfied. Since $\gamma^{*} \leq 0$ and $\gamma_{*}>-\frac{\hat{\Psi}_{*}}{r^{\tau}}$, we know that condition $\left(\mathrm{H}_{5}\right)$ holds.

Next, the existence condition $\left(\mathrm{H}_{3}\right)$ becomes

$$
\frac{r\left(\sigma r+\gamma_{*}\right)^{\tau}}{1+\left(r+\gamma^{*}\right)^{\tau+\eta}}>\Psi^{*}
$$

for some $r>0$. Since $\tau>0,0 \leq \eta<1$ and $\gamma^{*} \leq 0$, we can choose $r>0$ large enough such that (3.13) is satisfied. 


\section{Existence results (II)}

In this section, we establish the existence of positive periodic solutions for the fourthorder differential equation (1.3) by using Schauder's fixed point theorem, which can be found in [22] (see p.61).

Lemma 4.1 ([22], see p.61) A compact operator $A: M \rightarrow M$ has a fixed point provided $M$ is a bounded, closed, convex, nonempty subset of a Banach space $X$ over $\mathbb{R}$.

Theorem 4.1 Assume that $\rho<\frac{\pi}{\omega}$ and $\left(\mathrm{H}_{1}\right)-\left(\mathrm{H}_{2}\right)$ hold. Furthermore, assume that the following condition holds:

$\left(\mathrm{H}_{3}^{\prime}\right)$ There exists a positive constant $R>0$ such that $R>\left(\Phi_{R}\right)_{*}+\gamma_{*}>0$ and

$$
R \geq g\left(\left(\Phi_{R}\right)_{*}+\gamma_{*}\right)\left(1+\frac{h(R)}{g(R)}\right) K^{*}+\gamma^{*}
$$

where $\Phi_{R}(t)=\int_{0}^{\omega} G(t, s) \phi_{R}(s) d s$.

Then (1.3) has at least one positive periodic solution.

Proof An $\omega$-periodic solution of (1.3) is just a fixed point of the map $T^{*}: X \rightarrow X$ defined by

$$
\left(T^{*} x\right)(t)=\int_{0}^{\omega} G(t, s)[f(s, x(s))+e(s)] d s=\int_{0}^{\omega} G(t, s) f(s, x(s)) d s+\gamma(t) .
$$

Let $R$ be a positive constant satisfying $\left(\mathrm{H}_{3}^{\prime}\right)$ and

$$
r=\left(\Phi_{R}\right)_{*}+\gamma_{*}
$$

Then we have $R>r>0$. Now we define the set

$$
\Omega=\{x \in X: r \leq x(t) \leq R \text { for all } t\} .
$$

Obviously, $\Omega$ is a closed convex set. Next we prove $T^{*}(\Omega) \subset \Omega$.

In fact, for each $x \in \Omega$ and for all $t \in[0, \omega]$, using the fact that $G(t, s)>0$ for all $(t, s) \in$ $[0, \omega] \times[0, \omega]$, together with condition $\left(\mathrm{H}_{1}\right)$, we get

$$
\begin{aligned}
\left(T^{*} x\right)(t) & =\int_{0}^{\omega} G(t, s) f(s, x(s)) d s+\gamma(t) \\
& =\int_{0}^{\omega} G(t, s) f^{+}(s, x(s)) d s+\gamma(t) \\
& \geq \int_{0}^{\omega} G(t, s) \phi_{R}(s) d s+\gamma(t) \\
& \geq\left(\Phi_{R}\right)_{*}+\gamma_{*}:=r>0,
\end{aligned}
$$

where $f^{+}(t, x)=\max \{0, f(t, x)\}$. 
On the other hand, by conditions $\left(\mathrm{H}_{2}\right)$ and $\left(\mathrm{H}_{3}^{\prime}\right)$, we have

$$
\begin{aligned}
\left(T^{*} x\right)(t) & =\int_{0}^{\omega} G(t, s) f(s, x(s)) d s+\gamma(t) \\
& =\int_{0}^{\omega} G(t, s) f^{+}(s, x(s)) d s+\gamma(t) \\
& \leq \int_{0}^{\omega} G(t, s) k(s)(g(x(s))+h(x(s))) d s+\gamma(t) \\
& \leq g(r)\left(1+\frac{h(R)}{g(R)}\right) K^{*}+\gamma^{*} \leq R .
\end{aligned}
$$

In conclusion, $T(\Omega) \subset \Omega$. Say $W$ is any bounded subset in $\Omega$. Then, for $\forall x \in W$, we have

$$
\begin{aligned}
\left\|T^{*} x\right\| & =\max _{t}\left|\int_{0}^{\omega} G(t, s) f(s, x(s)) d s+\gamma(t)\right| \\
& \leq \max _{t}\left|\int_{0}^{\omega} G(t, s) f(s, x(s)) d s\right|+|\gamma|^{*} \\
& \leq M\left|f_{R}\right|_{1}+|\gamma|^{*}:=N_{3},
\end{aligned}
$$

where $|\gamma|^{*}=\max _{t \in[0, \omega]}|\gamma(t)|,\left|f_{R}\right|=\max _{r \leq x(t) \leq R}|f(t, x(t))|,\left|f_{R}\right|_{1}=\int_{0}^{\omega}\left|f_{R}\right| d t=\omega\left|f_{R}\right|$.

Due to the continuity of $G$, we have

$$
\begin{aligned}
\left|\frac{d T^{*} x}{d t}\right| & =\left|\int_{0}^{\omega} \frac{\partial G(t, s)}{\partial t}[f(s, x(s))+e(s)] d s\right| \\
& \leq \int_{0}^{\omega}\left|\frac{\partial G(t, s)}{\partial t}\right| f(s, x(s))+e(s) \mid d s \\
& \leq B^{\prime}\left(\left|f_{R}\right|_{1}+|e|_{1}\right):=N_{1},
\end{aligned}
$$

where $B^{\prime}=\max \left|\frac{\partial G(t, s)}{\partial t}\right|$ for all $(t, s) \in[0, \omega] \times[0, \omega],|e|_{1}=\int_{0}^{\omega}|e(s)| d s$.

Therefore, using the Arzela-Ascoli theorem, it is easy to show that $T^{*}$ is compact in $\Omega$. Hence, the proof is finished by Schauder's fixed point theorem.

As an application of Theorem 4.1, we consider the case $\gamma_{*}=0$. The following theorem is a direct result of Theorem 4.1.

Theorem 4.2 Assume that $\rho<\frac{\pi}{\omega}$ holds and $f(t, x)$ satisfies conditions $\left(\mathrm{H}_{1}\right)$ and $\left(\mathrm{H}_{2}\right)$. Furthermore, assume that the following condition holds:

$\left(\mathrm{H}_{3}^{\prime \prime}\right)$ There exists a positive constant $R>0$ such that $R>\left(\Phi_{R}\right)_{*}$ and

$$
g\left(\left(\Phi_{R}\right)_{*}\right)\left(1+\frac{h(R)}{g(R)}\right) K^{*}+\gamma^{*} \leq R
$$

If $\gamma_{*}=0$, then (1.3) has at least one positive periodic solution.

Corollary 4.1 Assume that $\rho<\frac{\pi}{\omega}$ holds. Assume that the following condition holds: 
$\left(\mathrm{F}_{3}\right)$ There exist continuous functions $d(t), \hat{d}(t) \succ 0$ and $0<\tau<1$ satisfying

$$
0 \leq \frac{\hat{d}(t)}{x^{\tau}} \leq f(t, x) \leq \frac{d(t)}{x^{\tau}} \quad \text { for all } x>0 \text { and a.e.t. }
$$

If $\gamma_{*}=0$, then (1.3) has at least one positive periodic solution.

Proof We will apply Theorem 4.2. We take

$$
\phi_{L}(t)=\frac{\hat{d}(t)}{L^{\tau}}, \quad k(t)=d(t), \quad g(x)=\frac{1}{x^{\tau}}, \quad h(x)=0 .
$$

Then conditions $\left(\mathrm{H}_{1}\right)$ and $\left(\mathrm{H}_{2}\right)$ are satisfied and the existence condition $\left(\mathrm{H}_{3}^{\prime \prime}\right)$ becomes

$$
R>\frac{\hat{\Psi}_{*}}{R^{\tau}}=r, \quad\left(\frac{R^{\tau}}{\hat{\Psi}_{*}}\right)^{\tau} \Psi^{*}+\gamma^{*} \leq R,
$$

where $\hat{\Psi}=\int_{0}^{\omega} G(t, s) \hat{d}(t) d t, \Psi=\int_{0}^{\omega} G(t, s) d(t) d t$ for some $R>0$. Note that $\Psi_{*}>0$, since $0<\tau<1$, we can choose $R>0$ large enough so that (4.3) is satisfied and the proof is complete.

Corollary 4.2 Assume that $\rho<\frac{\pi}{\omega}$ holds. Assume that the following condition holds:

$\left(\mathrm{F}_{4}\right)$ There exist continuous functions $d(t), \hat{d}(t) \succ 0$ and $0<\tau<1,0 \leq \eta<1$ satisfying

$$
0 \leq \frac{\hat{d}(t)}{x^{\tau}} \leq f(t, x) \leq \frac{d(t)}{x^{\tau}}+d(t) x^{\eta} \quad \text { for all } x>0 \text { and a.e. } t .
$$

If $\gamma_{*}=0$, then (1.3) has at least one positive periodic solution.

Proof We will apply Theorem 4.2. We take

$$
\phi_{L}(t)=\frac{\hat{d}(t)}{L^{\tau}}, \quad k(t)=d(t), \quad g(x)=\frac{1}{x^{\tau}}, \quad h(x)=x^{\eta} .
$$

Then conditions $\left(\mathrm{H}_{1}\right)$ and $\left(\mathrm{H}_{2}\right)$ are satisfied and the existence condition $\left(\mathrm{H}_{3}^{\prime \prime}\right)$ becomes

$$
R>\frac{\hat{\Psi}_{*}}{R^{\tau}}=r, \quad\left(\left(\frac{R^{\tau}}{\hat{\Psi}_{*}}\right)^{\tau}+R^{\eta}\right) \Psi^{*}+\gamma^{*} \leq R
$$

for some $R>0$. So, we can choose $R>0$ large enough so that (4.4) is satisfied and the proof is complete.

The next results explore the case when $\gamma_{*}>0$.

Theorem 4.3 Assume that $\rho<\frac{\pi}{\omega}$ holds and $f(t, x)$ satisfies $\left(\mathrm{H}_{2}\right)$. Furthermore, assume that the following condition holds:

$\left(\mathrm{H}_{3}^{\prime \prime \prime}\right)$ There exists $R>0$ such that

$$
g\left(\gamma_{*}\right)\left(1+\frac{h(R)}{g(R)}\right) K^{*}+\gamma^{*} \leq R
$$

If $\gamma_{*}>0$, then (1.3) has at least one positive periodic solution. 
Proof We follow the same strategy and notation as in the proof of Theorem 4.1. Let $R$ be the positive constant satisfying $\left(\mathrm{H}_{3}^{\prime \prime \prime}\right)$ and let $r=\gamma_{*}$; then $R>r>0$ since $R>\gamma^{*}$. Next we prove that $T^{*}(\Omega) \subset \Omega$.

For each $x \in \Omega$ and for all $t \in[0, \omega]$, by the non-negative sign of $G^{11}(t, s)$ and $f(t, x)$, we have

$$
\begin{aligned}
\left(T^{*} x\right)(t) & =\int_{0}^{\omega} G(t, s) f(s, x(s)) d s+\gamma(t) \\
& =\int_{0}^{\omega} G(t, s) f^{+}(s, x(s)) d s+\gamma(t) \\
& \geq \gamma_{*}=r>0 .
\end{aligned}
$$

On the other hand, by $\left(\mathrm{H}_{2}\right)$ and $\left(\mathrm{H}_{3}^{\prime \prime \prime}\right)$, we have

$$
\begin{aligned}
\left(T^{*} x\right)(t) & =\int_{0}^{\omega} G(t, s) f(s, x(s)) d s+\gamma(t) \\
& =\int_{0}^{\omega} G(t, s) f^{+}(s, x(s)) d s+\gamma(t) \\
& \leq \int_{0}^{\omega} G(t, s) k(s)(g(x(s))+h(x(s))) d s+\gamma(t) \\
& \leq\left(g(r)\left(1+\frac{h(R)}{g(R)}\right)\right) K^{*}+\gamma^{*} \\
& \leq R .
\end{aligned}
$$

In conclusion, $T^{*}(\Omega) \subset \Omega$. Meanwhile, using the Arzela-Ascoli theorem, it is easy to show that $T^{*}$ is compact in $\Omega$. Therefore, by Schauder's fixed point theorem, our result is proven.

Corollary 4.3 Assume that $\rho<\frac{\pi}{\omega}$ holds. Assume that the following condition holds:

$\left(\mathrm{F}_{5}\right)$ There exist a continuous function $d(t) \succ 0$ and a constant $\tau>0$ satisfying

$$
0 \leq f(t, x) \leq \frac{d(t)}{x^{\tau}} \quad \text { for all } x>0 \text { and a.e.t. }
$$

If $\gamma_{*}>0$, then (1.3) has at least one positive periodic solution.

Proof We apply Theorem 4.3. We take

$$
k(t)=d(t), \quad g(x)=\frac{1}{x^{\tau}}, \quad h(x)=0 .
$$

Then condition $\left(\mathrm{H}_{2}\right)$ is satisfied and the existence condition $\left(\mathrm{H}_{3}^{\prime \prime \prime}\right)$ is also satisfied if we take $R>0$ with

$$
R \geq \frac{\Psi^{*}}{\gamma_{*}^{\tau}}+\gamma^{*}
$$

Corollary 4.4 Assume that $\rho<\frac{\pi}{\omega}$ and $\left(\mathrm{F}_{2}\right)$ hold. If $\gamma_{*}>0$, then (1.3) has at least one positive periodic solution. 
Proof We apply Theorem 4.3. We take

$$
k(t)=d(t), \quad g(x)=\frac{1}{x^{\tau}}, \quad h(x)=x^{\eta} .
$$

Then condition $\left(\mathrm{H}_{2}\right)$ is satisfied and the existence condition $\left(\mathrm{H}_{3}^{\prime \prime \prime}\right)$ is also satisfied if we take $R>0$ with

$$
R \geq \Psi^{*}\left(\frac{1}{\gamma_{*}^{\tau}}+R^{\eta}\right)+\gamma^{*}
$$

On the other hand, condition $\left(\mathrm{H}_{2}\right)$ implies in particular that the nonlinearity $f(t, x)$ is non-negative for all values $(t, x)$, which is quite a hard restriction. In the following, we show how to avoid this restriction for $\gamma_{*}>0$.

Theorem 4.4 Assume that $\rho<\frac{\pi}{\omega}$ holds. Furthermore, assume that the following conditions hold:

$\left(\mathrm{H}_{2}^{*}\right)$ There exist continuous, non-negative functions $g(x)$ and $k(t)$ such that

$$
f(t, x) \leq k(t) g(x) \quad \text { for all }(t, x) \in[0, \omega] \times(0, \infty)
$$

and $g(x)>0$ is non-increasing in $x \in(0, \infty)$.

$\left(\mathrm{H}_{3}^{*}\right)$ Let us define

$$
R:=g\left(\gamma_{*}\right) K^{*}+\gamma^{*}
$$

and assume that $f(t, x) \geq 0$ for all $(t, x) \in[0, \omega] \times(0, R]$.

If $\gamma_{*}>0$, then (1.3) has at least one positive periodic solution.

Proof We again use Schauder's fixed point theorem. Let $R$ be the positive constant satisfying $\left(\mathrm{H}_{3}^{*}\right)$ and $r=\gamma_{*}$; then $R>r>0$ since $R>\gamma^{*}$. By again using the method of Theorem 4.3, it is easy to prove that $T^{*}(\Omega) \subset \Omega$. We omit the details.

Corollary 4.5 Assume that $\rho<\frac{\pi}{\omega}$ holds. Assume that the following condition holds:

$\left(_{6}\right)$ There exist constants $\tau, \eta, \mu>0$ satisfying

$$
f(t, x)=\frac{1}{x^{\tau}}-\mu x^{\eta} \quad \text { for all } x>0 \text { and a.e. } t .
$$

If $\gamma_{*}>0$, then (1.3) has at least one positive periodic solution for each $0 \leq \mu<\mu^{\prime}$, where $\mu^{\prime}$ is some positive constant.

Proof The nonlinearity is

$$
f(t, x)=\frac{1}{x^{\tau}}-\mu x^{\eta},
$$

and therefore $\left(\mathrm{H}_{2}^{*}\right)$ holds with $k(t)=1, g(x)=\frac{1}{x^{\tau}}$. Let $\Upsilon(t)=\int_{0}^{\omega} G(t, s) d s$. Then $R$ as defined in $\left(\mathrm{H}_{3}^{*}\right)$ is just $R=\frac{\Upsilon^{*}}{\gamma_{*}^{\tau}}+\gamma^{*}$. Note that $f(t, x) \geq 0$ if and only if $x^{\tau+\eta} \leq 1 / \mu$. Therefore, $\left(\mathrm{H}_{3}^{*}\right)$ 
is verified for any $\mu<R^{-(\tau+\eta)}$. As a consequence, the result holds for

$$
\mu^{\prime}=\left(\frac{\Upsilon^{*}}{\gamma_{*}^{\tau}}+\gamma^{*}\right)^{-(\tau+\eta)}
$$

Finally, we consider $\gamma^{*} \leq 0$.

Theorem 4.5 Assume that $\rho<\frac{\pi}{\omega}$ and $\left(\mathrm{F}_{3}\right)$ hold. If $\gamma^{*} \leq 0$ and

$$
\gamma_{*} \geq\left[\frac{\hat{\Psi}_{*}}{\left(\Psi^{*}\right)^{\tau}} \tau^{2}\right]^{\frac{1}{1-\tau^{2}}}\left(1-\frac{1}{\tau^{2}}\right),
$$

then there exists a positive $\omega$-periodic solution of (1.3).

Proof In this case, to prove that $T^{*}(\Omega) \subset \Omega$ defined by (4.2), it is sufficient to find $0<r<R$ such that

$$
\frac{\hat{\Psi}_{*}}{R^{\tau}}+\gamma_{*} \geq r, \quad \frac{\Psi^{*}}{r^{\tau}} \leq R .
$$

Taking $R=\frac{\Psi^{*}}{r^{\tau}}$, the first inequality holds if $r$ verifies

$$
\frac{\hat{\Psi}_{*}}{\left(\Psi^{*}\right)^{\tau}} r^{\tau^{2}}+\gamma_{*} \geq r
$$

or equivalently,

$$
\gamma_{*} \geq f(r):=r-\frac{\hat{\Psi}_{*}}{\left(\Psi^{*}\right)^{\tau}} \tau^{\tau^{2}}
$$

The function $f(r)$ possesses a minimum at $r_{0}:=\left[\frac{\hat{\Psi}_{*}}{\left(\Psi^{*}\right)^{\tau}} \tau^{2}\right]^{\frac{1}{1-\tau^{2}}}$. Let $r=r_{0}$. Then the first inequality holds in (4.6) if $\gamma_{*} \geq f\left(r_{0}\right)$, which is just condition (4.5). The second inequality holds directly by the choice of $R$, and it would remain to prove that $R=\frac{\Psi^{*}}{r_{0}^{\tau}}>r_{0}$. This is easily verified through elementary computations. Using the Arzela-Ascoli theorem, it is easy to show that $T^{*}$ is compact in $\Omega$. Therefore, the proof is completed by Schauder's fixed point theorem.

\section{Competing interests}

The authors declare that they have no competing interests.

Authors' contributions

YX and ZC worked together in the derivation of the mathematical results. Both authors read and approved the final manuscript.

\section{Author details}

${ }^{1}$ College of Computer Science and Technology, Henan Polytechnic University, Jiaozuo, 454000, China. ${ }^{2}$ School of Mathematics and Information Science, Henan Polytechnic University, Jiaozuo, 454000, China.

\section{Acknowledgements}

$Y X$ and ZC would like to thank the referee for invaluable comments and insightful suggestions. This work was supported by NSFC project (Nos. 11501170, 11271339) and the Fundamental Research Funds for the Universities of Henan Province (NSFRF140142). 


\section{References}

1. Ding, TR: A boundary value problem for the periodic Brillouin focusing system. Acta Sci. Natur. Univ. Pekinensis 11, 31-38 (1965)

2. Taliaferro, S: A nonlinear singular boundary value problem. Nonlinear Anal. TMA 3, 897-904 (1979)

3. Bonheure, D, De Coster, C: Forced singular oscillators and the method of lower and upper solutions. Topol. Methods Nonlinear Anal. 22, 927-938 (2003)

4. Rachunková, I, Tvrdý, M, Vrkoč, I: Existence of nonnegative and nonpositive solutions for second order periodic boundary value problems. J. Differ. Equ. 176, 445-469 (2001)

5. Cheng, ZB, Ren, JL: Periodic and subharmonic solutions for Duffing equation with singularity. Discrete Contin. Dyn. Syst., Ser. A 32, 1557-1574 (2012)

6. Fonda, A, Manásevich, R, Zanolin, F: Subharmonic solutions for some second order differential equations with singularities. SIAM J. Math. Anal. 24, 1294-1311 (1993)

7. Xia, J, Wang, ZH: Existence and multiplicity of periodic solutions for the Duffing equation with singularity. Proc. R. Soc. Edinb., Sect. A 137, 625-645 (2007)

8. Zhang, MR: Periodic solutions of Liénard equations with singular forces of repulsive type. J. Math. Anal. Appl. 203 254-269 (1996)

9. Zhang, MR: A relationship between the periodic and the Dirichlet BVPs of singular differential equations. Proc. R. Soc. Edinb., Sect. A 128, 1099-1114 (1998)

10. Torres, P: Weak singularities may help periodic solutions to exist. J. Differ. Equ. 232, 277-284 (2007)

11. Chu, JF, Torres, P, Zhang, MR: Periodic solution of second order non-autonomous singular dynamical systems. J. Differ. Equ. 239, 196-212 (2007)

12. Wang, HY: Positive periodic solutions of singular systems with a parameter. J. Differ. Equ. 249, 2986-3002 (2010)

13. Sun, J, Liu, Y: Multiple positive solutions of singular third-order periodic boundary value problem. Acta Math. Sci. 25 81-88 (2005)

14. Cheng, ZB, Ren, JL: Periodic solutions for a fourth-order Rayleigh type $p$-Laplacian delay equation. Nonlinear Anal. TMA 70, 516-523 (2009)

15. Conti, M, Terracini, S, Verzini, G: Infinitely many solutions to fourth-order superlinear periodic problem. Trans. Am. Math. Soc. 356, 3283-3300 (2003)

16. Han, GD, Xu, ZB: Multiple solutions of some nonlinear fourth-order beam equations. Nonlinear Anal. TMA 68 3646-3656 (2008)

17. Li, W, Zhang, MR: Non-degeneracy and uniqueness of periodic solutions for some superlinear beam equations. Appl. Math. Lett. 70, 516-523 (2009)

18. Ma, RY, Dai, GW: Periodic solutions of nonlocal semilinear fourth-order differential equations. Nonlinear Anal. TMA 74 5023-5029 (2011)

19. Mosconi, S, Santra, S: On the existence and non-existence of bounded solutions for a fourth-order ODE. J. Differ. Equ. 255, 4149-4168 (2013)

20. Zhao, $\mathrm{CH}$, Chen, W, Zhou, JL: Periodic solutions for a class of fourth-order nonlinear differential equations. Nonlinear Anal. TMA 72, 1221-1226 (2010)

21. Agarwal, RP, O'Regan, D, Wong, PJY: Positive Solutions of Differential, Difference and Integral Equations. Kluwer Academic, Dordrecht (1999)

22. Zeidler, E: Applied Functional Analysis. Applied Mathematical Sciences, vol. 108. Springer, New York (1995)

\section{Submit your manuscript to a SpringerOpen ${ }^{\circ}$ journal and benefit from:}

- Convenient online submission

- Rigorous peer review

Immediate publication on acceptance

- Open access: articles freely available online

- High visibility within the field

- Retaining the copyright to your article 